\title{
Funcionamiento de las políticas gubernamentales para la prevención y el control del dengue: el caso de Arauca y Armenia en Colombia
}

\author{
Ligia Constanza Velásquez, Juliana Quintero, Tatiana García-Betancourt, \\ Catalina González-Uribe, Mauricio Fuentes-Vallejo \\ Centro de Estudios e Investigación en Salud, Fundación Santa Fe de Bogotá, Bogotá, D. C., Colombia
}

\begin{abstract}
Introducción. El dengue es un problema de salud pública en aumento en las zonas urbanas, debido a que su transmisión está asociada con el ambiente y el comportamiento de las personas y las comunidades. El modelo propuesto por Ecosalud para su estudio, incluye el análisis del funcionamiento de las políticas gubernamentales.

Objetivo. Describir el funcionamiento de las políticas para prevenir y controlar el dengue en dos ciudades colombianas.

Materiales y métodos. Se adelantó un estudio cualitativo, para lo cual se recopiló información mediante entrevistas a funcionarios del sector de la salud y la educación, cuyos contenidos se procesaron en Ethnograph con base en las categorías de análisis propuestas en el modelo de 'implementación desde la base'.

Resultados. Se encontró que había desarticulación entre el departamento y el municipio, así como un desarrollo escaso del protocolo de dengue y dengue hemorrágico en cuanto a la participación comunitaria, las estrategias de comunicación y los estudios de conocimientos, actitudes y prácticas de los pobladores. La estrategia de gestión integrada se ve limitada por la inestabilidad del recurso humano, la escasa coordinación entre instituciones y sectores, y la falta de énfasis en las intervenciones de promoción tanto a nivel individual como de la población.

Conclusiones. En Arauca es necesario fortalecer la coordinación entre el departamento y el municipio. Tanto en Arauca como en Armenia se requiere mejorar la administración de los recursos humanos y la coordinación interinstitucional, así como fortalecer la promoción y la prevención, para lograr el efecto esperado sobre los factores determinantes del dengue.
\end{abstract}

Palabras clave: dengue/legislación y jurisprudencia, prevención de enfermedades transmisibles, control de enfermedades transmisibles.

doi: http://dx.doi.org/10.7705/biomedica.v35i2.2332

\section{Operation of government policies for the prevention and control of dengue: The cases of Arauca and Armenia}

Introduction: Dengue is a growing public health problem in urban areas, given that its transmission is associated with the environment, as well as with the behaviour of individuals and communities. The model proposed by Ecohealth to study this problem includes the operation of government policies.

Objective: To describe the operation of policies to prevent and control dengue in two Colombian cities. Materials and methods: A qualitative study was conducted. Data was collected through interviews with officials who worked in the health and education sectors. The contents were processed with the Ethnograph software, using the proposals of the bottom-up model of implementation as analytical categories.

Results: A lack of coordination was identified between department and municipal offices, with few developments of the protocol for dengue and severe dengue in terms of community participation, communication strategies and studies of inhabitants' knowledge, attitudes and practices. The integrated management strategy was limited by the instability of human resources, limited intra-institutional and cross-sectorial coordination and little emphasis on promotion interventions, both at the individual and population levels.

Conclusions: Coordination between the departament and the municipality needs to be strengthened in the city of Arauca. Both here and in Armenia administration of human resources and interinstitutional coordination should be improved. Promotion of preventative measures should be strengthened to impact on the determinants of dengue.

Key words: Dengue, legislation \& jurisprudence; communicable disease prevention; communicable disease control.

doi: http://dx.doi.org/10.7705/biomedica.v35i2.2332

Contribución de los autores:

Tatiana García-Betancourt y Mauricio Fuentes-Vallejo: recolección de datos

Todos los autores contribuyeron en la redacción del artículo. 
El dengue se considera un problema mundial de salud pública de gran importancia, el cual se manifiesta principalmente en contextos urbanos y se relaciona con la densidad de las poblaciones, tanto de mosquitos como de seres humanos (1). Es una enfermedad viral que se transmite a las personas mediante la picadura de la hembra del mosquito Aedes aegypti, lo cual puede suceder en los dormitorios, baños y cocinas de las viviendas humanas en donde se halla (1). La prevención y el control de la enfermedad se basan en la adopción de medidas eficaces contra la proliferación del mosquito.

En Colombia, el mosquito se ha adaptado a las zonas boscosas y a las viviendas. Los sitios en los que puede reproducirse son aquellos donde haya agua limpia estancada $y$, principalmente la almacenada en recipientes, en zonas urbanas o en entornos domésticos (1).

El control de la transmisión del dengue se centra tradicionalmente en intervenciones basadas en enfoques lineales, como el control por rociamiento de la reproducción del mosquito, y son pocos los intentos de abordarlo con una visión que involucre las dimensiones del ecosistema asociadas con la presencia del vector (2), por lo que este fenómeno no se ha analizado desde una perspectiva ecosistémica en que participen y converjan diversos actores e instituciones (3).

Dicha perspectiva metodológica es la que propone el método de Ecosalud para el estudio de problemas de salud como el dengue, con base en el análisis de los sistemas sociales y ecológicos del territorio considerado, teniendo en cuenta, entre otras, las dimensiones ecológica, social, cultural, económica y de gobernanza mediante procesos 'transdisciplinarios' (4). Dicho enfoque apunta a que el conocimiento producido por la investigación se transforme en acciones tendientes a modificar las condiciones preexistentes y a influir, entre otros ámbitos, en el de las políticas públicas, para lo cual es preciso analizar la política formal y la estructura legal creada para su funcionamiento, de manera

\footnotetext{
Correspondencia:

Juliana Quintero, Centro de Estudios e Investigación en Salud, Fundación Santa Fe de Bogotá, Carrera 7B No 123-90, tercer piso, Bogotá, D. C., Colombia

Teléfono: (571) 603 0303, extensión 5715; fax: (571) 2146668

juliana.quintero@fsfb.org.co

Recibido: 10/03/14; aceptado: 18/12/14
}

tal que puedan impulsarse cambios permanentes que garanticen la sostenibilidad futura de las intervenciones (5).

Bajo esta perspectiva se adelantó un estudio para analizar la proliferación de $A$. aegypti en las ciudades de Arauca y Armenia, el cual incluyó la descripción del funcionamiento de tres de las políticas gubernamentales para prevenir y controlar el dengue, a saber: la Ley 715 de 2001 (6), que asigna responsabilidades a los departamentos y municipios en este campo; el protocolo de dengue y dengue hemorrágico, que establece las directrices para los planes y programas e intervenciones en los territorios (7), y la estrategia de gestión integrada (8), la cual propone un modelo de fortalecimiento de los programas por medio de asociaciones encaminadas a lograr mejores resultados en la reducción de los factores de riesgo, así como de la vigilancia integral y el control de $A$. aegypti. Los resultados hacen parte de la línea de base que permitirá el diseño, implementación, evaluación e intensificación de las intervenciones para disminuir el dengue en estas ciudades.

\section{Materiales y métodos}

\section{Tipo de estudio}

Se hizo un estudio de corte transversal en cuyo marco se describió el funcionamiento de las políticas gubernamentales en estas ciudades, utilizando el modelo de la "implementación desde la base" desarrollado por Williams (9), el cual propone la "implementación de campo" como el ámbito en que toda política se consuma o se frustra, rescatando la importancia de la organización local y de los actores empíricos que prestan los servicios gubernamentales al público y que suelen ocupar los escalones más bajos de la administración. Se seleccionó este modelo por su coherencia con la perspectiva metodológica de Ecosalud, la cual también rescata el contexto local como el sitio de observación de un problema.

Este modelo propone, entre otras, las siguientes categorías de análisis: 1) la estructura organizacional, es decir, la dependencia responsable de dirigir y ejecutar las intervenciones; 2) los planes y programas, es decir, las estrategias y acciones programadas y ejecutadas en las intervenciones; 3) la comunicación entre las organizaciones, entendida como los mecanismos desarrollados por las organizaciones involucradas para coordinar la programación y el desarrollo de las actividades; 4) las restricciones, es decir, las condiciones 
económicas, políticas y sociales del entorno que limitan la ejecución de las actividades por parte de los diferentes actores, y 5) la actitud de los responsables del funcionamiento de las políticas, 0 sea, su capacidad y voluntad de ejecutarlas.

La descripción de este proceso puede abordarse desde dos componentes: la 'macroimplementación', que se refiere a la generación y cumplimiento de las condiciones necesarias para que una política pueda ponerse en práctica en un ámbito determinado de una localidad (10), y la 'microimplementación', que consiste en el conjunto de servicios concretos contemplado en una política, y que las organizaciones y agentes locales llevan a cabo con sus patrones particulares de gestión y rendimiento para atender a una población determinada (11).

En este contexto teórico se describió el funcionamiento de las políticas en Armenia y Arauca, sitios en donde se adelantó un diagnóstico del comportamiento de $A$. aegypti y cuya selección obedeció a los siguientes criterios: 1) la alta incidencia de dengue y dengue grave; entre 1999 y 2010; Armenia reportó 24.008 casos, con una tasa de incidencia promedio de 712,1 por 100.000 habitantes, posicionándose en el cuarto lugar a nivel nacional y el primero en la región centro-occidente, en tanto que, en el mismo periodo, Arauca, de particular interés por su carácter fronterizo, acumuló 7.992 casos, con una tasa de incidencia promedio de 954,1 por 100.000 habitantes, ubicándose en el puesto 14 a nivel nacional y en el segundo en su región (12); 2) el contar con programas operantes de control de vectores, y 3) la participación en los estudios para la prevención y el control de la malaria y el dengue en Colombia, llevados a cabo por el programa de investigación con enfoque ecosistémico en salud.

\section{Tamaño de la muestra}

Dada la necesidad de entrevistar a personas informadas dispuestas a hablar ampliamente con el investigador, se utilizó un muestreo teórico, también denominado intencionado (13), mediante el cual se seleccionaron 14 informantes (ocho en Arauca y seis en Armenia), a partir del universo de funcionarios o empleados que trabajaban en entidades de salud y educación en el sector público y en el privado, con base en los siguientes criterios de inclusión: que tuvieran relación con el funcionamiento de las políticas de prevención y control de la transmisión del dengue; que garantizaran la mayor cantidad y la mejor calidad de información posibles, y que aceptaran participar en el estudio. Bajo estas premisas, se incluyeron funcionarios de las secretarías de salud y educación, y de las entidades prestadoras de servicios, así como técnicos encargados de llevar a cabo las intervenciones, los cuales se consideraron como un universo único dado el propósito de describir el funcionamiento de las políticas a partir de la visión de quienes las implementan.

\section{Recolección de los datos}

Se hicieron entrevistas formales semiestructuradas, las cuales permitieron obtener información sobre los aspectos organizacionales, y registrar las opiniones y actitudes de los encargados de ejecutar las políticas. Se preparó un consentimiento informado en el que se explicaba el propósito del estudio y de la entrevista, su duración, la conveniencia de grabarla, el uso de la información que se extractara de su contenido y el nombre de la investigadora encargada de hacerla. Una vez se contactaron los informantes seleccionados en cada ciudad, se les solicitó suscribir este documento y se concertaron la fecha, el lugar y la hora de las entrevistas.

Las entrevistas estuvieron a cargo de una de las investigadoras, con base en preguntas orientadoras sobre los siguentes aspectos: 1) programas y actividades desarrollados para el control del dengue; 2) dependencias responsables de dirigirlas; 3) personas encargadas de ejecutar dichas actividades; 4) instituciones a cargo de su ejecución; 5) coordinación entre las organizaciones de las acciones de prevención y control del dengue ; 6) restricciones para la ejecución de las actividades, y 7) su opinión sobre los lineamientos nacionales para prevenir y controlar el dengue.

\section{Procesamiento y análisis de la información}

Las grabaciones de las entrevistas se transcribieron y guardaron en archivos digitales de Word, para luego exportalos al programa Ethnograph, el cual está diseñado para el análisis descriptivo e interpretativo de textos mediante la descontextualización y 'recontextualización' con base en las categorías previamente establecidas por el investigador que, en este caso, fueron las incluidas en el modelo de análisis ya descrito.

\section{Resultados}

\section{Funcionamiento de las políticas en Arauca}

En cuanto a su capacidad de gestión, el municipio de Arauca está clasificado en categoría 4 según lo establecido por la Ley 715 de 2001, es decir, que 
no cuenta con la certificación para administrar los recursos, por lo cual comparte con el departamento la gestión de las acciones de promoción, prevención, vigilancia y control de vectores y zoonosis (6). Por ello, entre los informantes clave entrevistados, se incluyeron a funcionarios del departamento y del municipio de Arauca.

Planes de gobierno. En su plan de desarrollo, y como parte del programa de salud pública, el departamento incluyó la meta de reducir en $20 \%$ la tasa de mortalidad de las enfermedades transmitidas por vectores, para lo cual identificaría y controlaría el $90 \%$ de los brotes de dengue mediante acciones adelantadas por el laboratorio departamental de salud pública. Además, se propuso realizar vigilancia y fortalecer el $90 \%$ de los espacios de participación social (14).

Si bien en el plan de desarrollo del municipio de Arauca se menciona la necesidad de mantener en cero la tasa de mortalidad por dengue, no se plantea una política específica para ello, sino que se propone el desarrollo de estrategias intersectoriales y de base comunitaria orientadas a promover la participación social para fortalecer los factores protectores, prevenir los factores de riesgo y controlar la transmisión de las enfermedades (15), entre ellas, el dengue. Esto se explica porque las intervenciones del municipio en este campo solo complementan las del departamento: "Quien maneja el programa es la Unidad Departamental de Salud, y la labor de la Secretaría de Salud en esa área no ha sido tan fuerte porque la Resolución 425 prioriza otras áreas, pero las acciones de prevención se desarrollan en las comunas más vulnerables donde hay más focos de infección con ETV" (funcionario de la Secretaría Municipal de Salud de Arauca entrevistado el 10 abril de 2013). Además de lo dicho por este funcionario, se estaban adelantando obras públicas para evitar el almacenamiento de aguas (15).

Planes, programas y actividades. La Unidad de Salud del departamento prioriza la vigilancia entomológica, el control químico, la vigilancia por laboratorio, las campañas y la comunicación social: "[...] adelantamos estudios de índices aédicos, información epidemiológica y de laboratorio acerca de serotipos y confirmaciones de casos, aplicación de larvicidas biológicos y químicos, control de epidemias, revisión de la atención de casos en los hospitales y estudios de campo cuando se dan casos positivos en los que se visita el domicilio de la persona y alrededor de la manzana y se buscan los pacientes que son febriles, remitiéndolos al sistema de salud. Además, se hacen jornadas de recolección de inservibles y uso de toldillos, cuñas de radio, afiches, pancartas [...]" (funcionario de la Unidad Administrativa Especial de Salud de Arauca entrevistado el 8 de abril de 2013).

La Secretaría Municipal de Salud solo desarrolla actividades para estimular la participación social como parte de otras estrategias: "[...] con la estrategia AIEPI se hace referencia a la prevención del dengue [...]" (funcionario de la Secretaría Municipal de Salud de Arauca entrevistado el 10 de abril de 2013).

La institución prestadora de servicios se encarga de notificar los casos y capacitar a sus usuarios: "[...] la enfermera o dos auxiliares se encargan de dictar las capacitaciones al personal que está esperando la atención y, apenas se diagnostica un caso como dengue, se llena su ficha epidemiológica y se notifica semanalmente por medio del Sivigila [...]" (enfermera de promoción y prevención de IPS entrevistada el 12 de abril de 2013 en Arauca). Dado que no es de carácter obligatorio, solo en algunos colegios los docentes del área de ciencias naturales y sus alumnos adelantan actividades puntuales en este sentido.

Estructura organizacional. La Unidad Administrativa Especial de Salud del departamento es la encargada de desarrollar las políticas y adelantar las intervenciones relacionadas con las enfermedades transmitidas por vectores. En el momento del estudio esta dependencia no contaba con personal de planta: "[...] Todos los funcionarios, desde el jefe operativo hasta los auxiliares, estamos tres meses y quince días [...]" (funcionario de la Unidad Administrativa Especial de Salud entrevistado el 8 de abril de 2013 en Arauca).

La Secretaría Municipal de Salud cuenta con un programa de salud pública cuyo objetivo es disminuir la incidencia de las enfermedades transmisibles, pero no existe un área específica que se encargue de la prevención y el control del dengue: "[...] por ser de cuarta categoría no tenemos ni el recurso ni podemos hacer actividades, pues no tenemos competencia. Si se presenta un caso de dengue, un auxiliar de enfermería y un técnico en salud pública hacen visita de campo y diligencian los formularios que vienen estipulados de la unidad de salud [...]" (funcionario de la Secretaría Municipal de Salud entrevistado el 10 de abril de 2013 en Arauca.) 
En la institución prestadora de servicios, la persona encargada de calidad en el momento del estudio era quien organizaba las actividades de "educación a los pacientes que llegan a las consultas" (enfermera de promoción y prevención de IPS entrevistada el 12 de abril de 2013 en Arauca).

Comunicación entre organizaciones. La Unidad Administrativa Especial de Salud del departamento es la encargada de este proceso en lo relacionado con la notificación de casos, el apoyo puntual de las alcaldías, la ayuda esporádica de los estudiantes en los colegios para la toma de registros y la educación a los padres de familia: "[...] todos los hospitales en todos los municipios mandan las fichas... Las alcaldías a veces nos suministran el combustible, prestan las volquetas para hacer recolección de inservibles... Los estudiantes van tomando los datos y nosotros vamos fumigando, y se dictan las charlas a los padres de familia y a los alumnos [...]" (funcionario de la Unidad Administrativa Especial de Salud para ETV entrevistado el 10 de abril de 2013).

En el municipio, la Secretaría de Salud es la encargada de coordinar las actividades educativas con las organizaciones comunitarias y, en cuanto a la notificación, la policía y el ejército informan sobre la situación de seguridad para adelantar los estudios de campo. Cuando se necesita apoyo puntual, la Secretaría Municipal debe acudir al departamento, por lo que es necesario fortalecer el vínculo con la Unidad Administrativa Especial de Salud: "[...] con los Comités de Participación Comunitaria y los Comités de Vigilancia Epidemiológica Comunitaria identificamos el dengue como prioridad y, como no tenemos competencia, hacemos la gestión en la Unidad para que envíen funcionarios..., deberíamos establecer una relación mucho más cercana con el departamento para que pudiéramos complementar las acciones [...]" (funcionario de la Secretaría Municipal de Salud entrevistado el 10 de abril de 2013 en Arauca).

En cuanto a la institución prestadora de servicios, la coordinación con las otras entidades es muy limitada y solo se hace para la vigilancia entomológica: "[...] se notifica al ente municipal y al ente departamental [...]" (enfermera de promoción y prevención de IPS entrevistada el 12 de abril de 2013 en Arauca). En los colegios se actúa únicamente cuando se encuentran casos: "[...] empiezan las campañas, pero no es nada constante
[...]" (funcionario de la Secretaría Municipal de Educación entrevistado el 10 de abril de 2013 en Arauca).

Restricciones para la operación de las políticas. Según los entrevistados, en el nivel departamental los principales obstáculos se asociaron con la asignación de recursos financieros y humanos: "[...] no escuchan la argumentación técnica para el manejo de los recursos..., montan otro que no tiene ni idea, como pueden llegar mejores pueden llegar malos [...]" (funcionario de la Unidad Administrativa Especial de Salud para ETV entrevistado el 10 de abril de 2013 en Arauca).

En el nivel municipal, los principales obstáculos identificados fueron las diferencias entre la planeación y la realidad de la situación, la desarticulación del sector y la falta de recursos financieros suficientes para emprender acciones: "[...] hay cosas que uno no las visualiza hasta que no entra al terreno..., contamos con quince millones de pesos y eso es insignificante para hacer una acción en una comunidad [...]" (funcionario de la Secretaría Municipal de Salud entrevistado el 10 de abril de 2013 en Arauca).

Las personas entrevistadas en la institución prestadora de servicios y en el sector educativo, consideraron que estas son actividades "extras" que aumentan la carga laboral de los empleados: "[...] no se cuenta con tiempo para parar la jornada para dictar una capacitación, siempre estoy atendiendo pacientes [...]" (enfermera de promoción y prevención de IPS entrevista el 12 de abril de 2013 en Arauca); "[...] si lo vas a poner un sábado o en jornadas contrarias, de cinco, tres dicen que no [...]" (funcionario de la Secretaría Municipal de Educación entrevistado el 10 de abril de 2013 en Arauca).

Actitud de los responsables del funcionamiento de las políticas. Si bien en el nivel nacional la estrategia de gestión integrada ha propiciado una mejor articulación entre la Dirección de Salud Pública del Ministerio de la Protección Social y el Instituto Nacional de Salud (16), en el departamento de Arauca no se ha oficializado su implementación: "[...] se hizo una sesión de trabajo, se socializó (sic.) y se le pidió al Departamento que empezara, pero no hay ningún documento [...]". En cuanto al componente de gerencia del programa, los entrevistados señalaron la necesidad de desarrollar la coordinación interinstitucional: "[...] abogacía (sic.) con educación, con servicios públicos para 
trabajar y dar un mejor impacto [...]", y en lo relativo al componente de prevención primaria, opinaron que el almacenamiento de agua era la principal barrera : "[...] argumentan que como el servicio de agua no es constante tienen que almacenar agua [...]" (funcionario de la Unidad Administrativa Especial de Salud entrevistado el 8 de abril de 2013 en Arauca).

En el nivel municipal, los entrevistados señalaron que la articulación interinstitucional contemplada en la estrategia de gestión integral, era difícil de lograr por la poca credibilidad de las instituciones: "[...] tienen campañas y campañas, pero los resultados son los mismos [...]" (funcionario del Colegio Santa Teresita entrevistado el 11 de abril de 2013 en Arauca); además, que la implementación del componente de promoción de la salud se veía entorpecido por la cultura y las costumbres de la comunidad, así como por su desmotivación y fatiga: "[...] al llanero le gustan las albercas grandes..., sienten que se han hecho cosas pero no se han visto los resultados [...]" (funcionario de la Secretaría Municipal de Salud entrevistado el 10 de abril de 2013 en Arauca).

\section{Funcionamiento de las políticas en Armenia}

A diferencia de la ciudad de Arauca, Armenia es un municipio de categoría 1, lo que le da a esta cuidad autonomía para manejar sus recursos, formular y ejecutar las acciones de promoción, prevención, vigilancia y control de $A$. aegypti, así como de impulsar los procesos de participación social (17).

Planes de gobierno. Para controlar la transmisión del dengue, el programa de salud pública hace vigilancia entomológica, en tanto que el programa de promoción social lleva a cabo acciones para estimular la participación social, y el programa de educación ambiental y ciudadana sostenible, consolida los colectivos socio-ambientales y la formación de promotores ambientales barriales. Además, a través del programa de infraestructura para el desarrollo se hace mantenimiento del espacio público y, con el programa de vivienda y hábitat, se garantizan entornos saludables (18).

Planes, programas y actividades. Para la vigilancia y control de vectores, la Secretaría de Salud recibe la información de las instituciones notificadoras y hace comprobación y seguimiento de los casos: "[...] verificamos que se cumplan los criterios, se hagan las pruebas para confirmar o descartar el caso y se hace una visita domiciliaria para confirmar la información con el paciente [...]"; además, se instalan ovitrampas: "[...] distribuidas en las plazas de mercado, el aeropuerto, la terminal de transporte, un 'servicentro' y una bomba de gasolina, tránsito de transporte de carga [...]". Para estimular la participación social se promueven los entornos saludables involucrando a los constructores: "[...] se está trabajando en hábitos y entornos saludables en viviendas, porque el modelo de construcción de sumideros y recámaras es altamente productivo en zancudo y son un criadero sin doliente [...]" (funcionario de la Secretaría Municipal de Salud entrevistado el 30 de enero de 2013 en Armenia). Además, los técnicos intervienen en las viviendas y sus alrededores: "[...] se hace la inspección de recipientes, criaderos potenciales de mosquitos..., en la vía pública, de los sumideros de agua lluvia y en lotes abandonados, la existencia de basuras..., se hace el levantamiento de los índices aédicos, y cuando tuvimos un brote localizado tuvimos que hacer intervención química [...]" (funcionario de la Secretaría Municipal de Salud entrevistado el 29 de enero del 2013 en Armenia).

Con apoyo del hospital se difunde información sobre estilos de vida protectores a través de medios electrónicos, “[...] utilizando tips y mensajes alusivos, mandando correos electrónicos a ciudadanos atendidos en las EPS [...]" (funcionario de la Secretaría Municipal de Salud entrevistado el 30 de enero de 2013 en Armenia), y "[...] entregamos folletos, damos educación, formación y comunicación en diferentes barrios, instituciones educativas y empresas [...]" (funcionario del Hospital del Sur entrevistado el 31 de enero de 2013 en Armenia). En los colegios se desarrollan actividades educativas y se revisan los depósitos de agua; además, se organizan "[...] conferencias a estudiantes y padres de familia, carteleras en las sedes educativas haciendo recomendaciones para el control del agua depositada e inspección a las instalaciones educativas para que no haya tanques - receptáculos donde se deposite agua que puedan ser generadores de la larva o del mosquito [...]" (funcionario de la Secretaría Municipal de Educación entrevistado el 30 de enero de 2013).

Estructura organizacional. La Secretaría Municipal de Salud cuenta con un grupo de zoonosis y vectores: "[...] un grupo funcional que se llama eventos de salud pública de origen animal [...]" (funcionario de la Secretaría Municipal de Salud entrevistado el 30 de enero de 2013 en Armenia). El hospital tiene un departamento de epidemiología 
que "[...] hace el monitoreo (sic.) de los conceptos técnicos y los diagnósticos de interés, la supervisión y la aplicación de la guía [...]" (funcionario del Hospital del Sur entrevistado el 31 de enero de 2013 en Armenia), y en la IPS "[...] la dirección médicocientífica es la encargada de hacer que se apliquen las guías y los protocolos de dengue [...]" (funcionario de la Clínica de la Sagrada Familia entrevistado el 31 de enero de 2013). Por último, la Secretaría de Educación tiene un funcionario que "[...] lidera, hace seguimiento y ofrece asistencia técnica a las instituciones educativas para los proyectos ambientales escolares y coordina las charlas de la Secretaría de Salud [...]", y en las instituciones educativas "[...] hay equipos de estudiantes que manejan los programas ambientales [...]" (funcionario de la Secretaría Municipal de Educación entrevistado el 30 de enero de 2013).

Comunicación entre organizaciones. La Secretaría Municipal de Salud interactúa con las entidades del sector de la salud en lo tocante a la notificación de casos, y con las juntas de acción comunal en lo relacionado con actividades puntuales: "[...] con las IPS, las EPS y el laboratorio departamental de salud pública hacemos una reunión de gestión del riesgo donde les contamos lo que está pasando con los eventos de interés en salud pública, entre ellos el dengue..., los presidentes permanentemente piden fumigaciones e intervenciones [...]" (funcionario de la Secretaría Municipal de Salud entrevistado el 30 de enero de 2013 en Armenia).

Restricciones para el funcionamiento de las políticas. Los funcionarios de la Secretaría Municipal de Salud entrevistados destacaron la carencia o la escasez de recursos financieros para la comunicación social masiva: "[...] para contratar comerciales radiales, incluso en televisión, para tener una estrategia de comunicación innovadora [...]' (funcionario de la Secretaría Municipal de Salud entrevistado el 30 de enero de 2013 en Armenia). Al igual que los entrevistados en la IPS, también consideraron como un obstáculo la resistencia de la comunidad a modificar sus creencias, actitudes y prácticas con relación al almacenamiento de agua, así como la falta de sentido de pertenencia que se generó después del terremoto: "[...] a pesar de que hemos estado tanto tiempo con la comunidad, no se han generado cambios de comportamiento en las personas..., la gente no cree que el zancudo se cría en el lavadero y cree que lavar el lavadero es vaciar agua que les cuesta plata... Cuando la gente viene de otras partes no tiene el enraizamiento de este 'pedacito es mío y yo lo cuido'; eso le ha hecho mucho daño a la ciudad [...]" (funcionario de la Secretaría Municipal de Salud entrevistado el 30 de enero de 2013 en Armenia). En palabras de otro entrevistado, "[...] los hábitos que tenemos como habitantes a veces no facilitan mucho el que las cosas se den [...]" (funcionario de la Clínica de la Sagrada Familia entrevistado el 31 de enero de 2013).

El personal operativo consideró que las intervenciones se ven limitadas por la insuficiencia de recursos humanos y la lentitud de los procesos: "[...] finalizando el año hay deficiencia en personal dedicado a la parte educativa con conocimiento de lo comunitario..., quisiera que fueran mucho más ágiles los trámites dentro de la administración para realizar las actividades y contratar personas o tener insumos [...]" (funcionario de la Secretaría Municipal de Salud entrevistado el 29 de enero de 2013 en Armenia).

Actitud de los responsables del funcionamiento de las políticas. Las personas de la Secretaría Municipal de Salud entrevistadas, expresaron su desacuerdo con la recomendación del Ministerio acerca de la aplicación del control químico después de un caso de mortalidad y estimaron inadecuada su relación con este: "[...] se debería tener una metodología que permitiera verificar índices de infestación y cuando sean altos hacer control químico preventivo..., se carece de una estrategia de comunicación permanente desde el nivel nacional..., que venga la gente del Ministerio más que a una auditoría a un acompañamiento [...]" (funcionario de la Secretaría Municipal de Salud entrevistado el 29 de enero de 2013 en Armenia).

Con relación a la implementación de la estrategia de gestión integrada, los funcionarios entrevistados afirmaron que la Secretaría carece de capacidad institucional en el componente de gerencia del programa, específicamente en lo concerniente al trabajo intersectorial, aspecto en el que Ministerio no ha mostrado mayores avances: "[...] no he podido ver el trabajo intersectorial ni siquiera desde el nivel nacional..., la estrategia plantea que hay que trabajar intersectorialmente, hacer intervenciones integrales y evitar la intervención química, pero cuando llegamos al pico máximo, entramos a fumigar porque la responsabilidad de la salud de los ciudadanos la tiene el Alcalde [...]" (funcionario de la Secretaría Municipal de Salud entrevistado el 30 de enero de 2013 en Armenia). A pesar de estas críticas, los entrevistados reconocen 
que la Secretaría ha desarrollado intervenciones con el sector educativo e intentado fortalecer el trabajo con otras entidades municipales: "[...] se han hecho trabajos de entornos saludables y programas de reciclaje, y existe el propósito de promover el trabajo con entidades como las Empresas Públicas de Armenia, los constructores, Planeación y Gobierno [...]" (funcionario de la Secretaría Municipal de Salud entrevistado el 30 de enero de 2013 en Armenia).

Por último, el personal operativo destacó la importancia de la participación social: "[...] ese paternalismo, asistencialismo, está mandado a recoger porque es costoso para el Estado, siendo responsabilidad de la misma comunidad, por intermedio de sus líderes, minimizar los problemas y la intervención de políticos [...]" (funcionario de la Secretaría Municipal de Salud entrevistado el 29 de enero de 2013 en Armenia).

\section{Discusión}

La implementación de una política social significa poner en operación, a través de una organización específica y de unos operadores concretos en una localidad determinada, un conjunto de acciones cuyas eventuales consecuencias benéficas para una población representan el logro de los objetivos ordenados por las políticas (9). En este contexto, en el presente estudio se describió, más no evaluó ni se comparó, la manera en que se han puesto en marcha tres de las políticas formuladas por la Nación para controlar y prevenir el dengue en las ciudades de Arauca y Armenia.

Con relación a la macroimplementación, Armenia cuenta con una estructura administrativa para asumir las competencias que en este asunto le asigna la Ley 715 de 2001 (6), así como el desarrollo de las intervenciones establecidas por el protocolo de dengue y dengue hemorrágico (7), y la implementación de la estrategia de gestión integrada (8). No es este el caso de Arauca, en donde los entrevistados consideraron que la ciudad no tiene ninguna responsabilidad en este campo diferente al apoyo puntual que debe darse al departamento, por lo que no se cuenta con una estructura específica. Las respuestas a la entrevista alertan sobre el rol de la ciudad en el control del dengue, teniendo en cuenta el compromiso de mejorar las condiciones de vida de sus pobladores y la obligatoriedad, en este campo, de un trabajo estrechamente coordinado con el departamento.

Ahora bien, en Armenia, los planes y programas gubernamentales reflejan la intención de disminuir la transmisión del dengue, lo cual guarda coherencia con la concepción del territorio como un ámbito en el que se articulan los procesos ambientales y sociales (18), y con la propuesta de Ecosalud en cuanto al papel del territorio en el que se presentan los problemas de salud (4).

En el momento del estudio, los planes y programas gubernamentales en Arauca reflejaban su desarticulación con el departamento, el cual centraba sus intervenciones en el control vectorial, en tanto que el municipio incluía la prevención y el control del dengue en la estrategia de atención integrada a las enfermedades prevalentes de la infancia (AIEPI). Esta situación sería adecuadad si las dos entidades territoriales estuvieran articuladas, lo que potenciaría sus efectos. Al no darse esta condición, estos se minimizan.

La comunicación entre organizaciones tanto en Armenia como en Arauca, se reveló muy débil, concentrada en las instituciones que conforman el sector de la salud, y principalmente relacionada con la notificación de casos y la organización de charlas educativas puntuales, lo que indica el poco poder de convocatoria de las secretarías de salud de estas dos ciudades y refleja una situación similar a la reportada por Rodríguez (18).

Con relación a la microimplementación de la política en Armenia en lo que tiene que ver con el protocolo de dengue y dengue hemorrágico (7), los hallazgos del estudio mostraron un gran desarrollo de la vigilancia entomológica y de salud pública, así como algunos avances en cuanto a la participación de entidades externas, como las del sector educativo, y un mayor involucramiento de los prestadores de servicios en temas de salud colectiva. Sin embargo, se destacó la carencia de información sobre los conocimientos, actitudes y prácticas de las comunidades, que pudiera explicar su resistencia al cambio, así como las tímidas acciones para estimular la participación social y las restricciones para utilizar la comunicación social.

En cuanto a la implementación de la estrategia de gestión integrada en Armenia, se encontró que el principal obstáculo para la gerencia del programa era el tipo de contratación del recurso humano, pues afecta la calidad y la continuidad de las intervenciones, así como los pocos avances del entonces Ministerio de Salud y Protección Social con respecto a la coordinación entre sectores. En el componente de inteligencia epidemiológica, se evidenciaron los avances, tal como lo ha reconocido el Ministerio de Salud al afirmar que la 
principal fortaleza de las direcciones territoriales de salud es la oportunidad de la notificación por parte de las unidades notificadoras (8). En cuanto a la gestión del conocimiento, llamó la atención que no se hubiera mencionado ningún avance. Por último, en lo concerniente a la promoción de la salud y la prevención primaria, se destacó la resistencia de la comunidad al cambio de comportamientos y la falta de sentido de pertenencia de los pobladores, lo que evidencia la necesidad imperiosa de hacer estudios sobre los conocimientos, actitudes y prácticas, que ayuden en el diseño de intervenciones efectivas.

La microimplementación de la política en la ciudad de Arauca se mostró desarticulada de la del departamento, ente que prioriza el desarrollo de la vigilancia entomológica y por el laboratorio, en detrimento de las intervenciones asociadas con la vinculación de la comunidad, el uso de medios masivos de comunicación social y los estudios sobre conocimientos, actitudes y prácticas de la población, hallazgos que concuerdan con lo reportado por Rodríguez (18) acerca de la falta de integración dentro y entre los sectores, así como de participación comunitaria. Asimismo, en la perspectiva de Ecosalud, este hallazgo señala la necesidad de fortalecer estos componentes en los proyectos (6). Reiteramos que el rol de la ciudad de Arauca es absolutamente marginal debido a que no se ha apropiado de las acciones de prevención y control del dengue, actitud que también se evidenció en la institución prestadora de servicios $y$ en el sector educativo.

Con relación a la estrategia de gestión integrada, quedó claro que la gerencia del programa de la Unidad de Salud del departamento enfrenta también los problemas ocasionados por la temporalidad e inestabilidad del recurso humano. En el componente de inteligencia epidemiológica, los hallazgos del estudio mostraron avances destacables en el departamento, pero no así en el municipio. Al igual que en la ciudad de Armenia, tampoco se evidenciaron avances en la gestión del conocimiento, en tanto que en el campo de la promoción de la salud, el departamento tiene debilidades en la integración con otras entidades. Los resultados del estudio muestran que las intervenciones del municipio son muy puntuales, situación que podría mejorarse si se diseñaran intervenciones bajo el modelo de Ecosalud, que expresamente propone integrar todas las dimensiones del ecosistema en donde se presenta el dengue (5).
A la luz de estos hallazgos, resultan pertinentes las siguientes recomendaciones.

- En la ciudad de Arauca sería necesario impulsar y fortalecer la coordinación con el departamento para evitar la desarticulación detectada, la cual limita el impacto de las intervenciones y el uso óptimo de los recursos.

- En las dos ciudades sería necesario desplegar y fortalecer la participación de la comunidad, así como estrategias de comunicación y la investigación sobre los conocimientos, actitudes y prácticas de los pobladores.

- Para impulsar la implementación de la estrategia de gestión integrada, en las dos ciudades se requeriría garantizar las condiciones para una adecuada gerencia del programa en términos de la estabilidad del personal, y fortalecer la coordinación entre las instituciones externas al sector en torno a la planeación y operación de las intervenciones, comprometiendo a las autoridades y a los actores locales interesados, con el fin de optimizar los recursos, aglutinar los esfuerzos y desarrollar las acciones estratégicas. Igualmente, se requeriría mayor énfasis en las intervenciones de promoción y fortalecimiento de la participación social.

- Sería necesario, igualmente, que el nivel nacional y los proyectos, desplieguen a profundidad la gestión del conocimiento y la prevención primaria del dengue.

Por último, es necesario mencionar que en este estudio no se incluyó la descripción de los recursos financieros porque, a pesar de ser elementos importantes de una adecuada operación, el análisis se centraba en la manera en que el trabajo se lleva a cabo en estas ciudades.

\section{Agradecimientos}

A Luis Bernardo Mejía, del Departamento de Ciencias Políticas de la Universidad de los Andes, por sus valiosos comentarios sobre este artículo.

\section{Conflicto de intereses}

Los autores declaramos que no tenemos intereses económicos o personales relativos a los resultados publicados en este artículo.

\section{Financiación}

Este estudio fue financiado con recursos de Colciencias asignados al proyecto "Enfoque ecosistémico para el diseño e implementación de estrategias sostenibles para dengue en las regiones de Arauca y Quindío" en el año 2012. 


\section{Referencias}

1. Ministerio de la Protección Social, Instituto Nacional de Salud, Organización Panamericana de la Salud. Gestión para la vigilancia entomológica y control del dengue. Fecha de consulta: 15 de noviembre de 2013. Disponible en: http://www.ins.gov.co/temas-de-interes/Dengue/03\%20 Vigilancia\%20entomo\%20dengue.pdf.

2. Vora N. Impact of antropogenic environmental alterations on vector-borne diseases. Medscap J Med. 2008;10:238.

3. Lebel J. Health: An ecosystem approach. Otawa: Canada International Development Research Centre; 2003.

4. Forget G, Lebel J. An ecosystem approach to human health. Int J Occup Environ Health. 2001:7(Suppl.2):S3-38.

5. Charron DF. Ecohealth: Origins and approach. En: Ecohealth research in practice. New York: Springer Link; 2012. p. 1-30. 10.1007/978-1-4614-0517-7

6. Congreso de la República. Ley 715. 2001. Fecha de consulta: 15 de noviembre de 2013. Disponible en: http:// www.minsalud.gov.co/Normatividad_Nuevo/LEY\%20 0715\%20DE\%202001.pdf.

7. Ministerio de la Protección Social, Instituto Nacional de Salud. Protocolo de dengue y dengue hemorrágico. Fecha de consulta: 15 de noviembre de 2013. Disponible en: http:// blog.utp.edu.co/maternoinfantil/files/2010/08/DengueEpidemiologia.pdf.

8. Ministerio de Salud, Organización Panamericana de la Salud. EGI 2012-2021 Estrategia de Gestión Integrada para la promoción, prevención y control de las Enfermedades Transmitidas por Vectores en Colombia, 2012-2021. Bogotá, marzo 26 de 2013. Fecha de consulta: 10 de noviembre de 2013. Disponible en: http://www.proyectomalariacolombia.co/ files/msps/EGIETVNacionalversion26demarzode2013.pdf.

9. Aguilar LF. Estudio introductorio. En: La implementación de las políticas. México: Miguel Ángel Porrúa Grupo Editorial; 1996. p.81.
10. Morales BF. Desarrollo regional sustentable: una reflexión desde las políticas públicas. Revista Digital Universitaria. 2003;6. Fecha de consulta: 3 enero de 2014. Disponible en: http://www.revista.unam.mx/vol.4/num6/art14/art14.htm.

11. Berman P. El estudio de la macro y micro implementación. En: Aguilar L, editor. La implementación de las políticas. México: Miguel Ángel Porrúa; 1996. p. 281-321.

12. Padilla JC, Rojas DP, Sáenz-Gómez R. Dengue en Colombia: epidemiología de la reemergencia a la hiperendemia. Bogotá D.C.; Guías de Impresión Limitada; 2012. p.80-3.

13. Salamanca AB, Martín C. El muestreo en la investigación cualitativa. Nure Investigación. 2007;27:1-4.

14. Gobernación de Arauca. Plan de Desarrollo Departamental 2012-2015. Es hora de resultados. Fecha de consulta: 1 de diciembre de 2013. Disponible en: http://www.arauca.gov. co/documentos/ORDENANZA\%20001E\%20PLAN DE DESARROLLO_DEPARTAMENTAL_2012-2015\%200K.pdf.

15. Municipio de Arauca. Plan de Desarrollo Municipal 20122015. Trabajo, progreso y solidaridad. Fecha de consulta: 15 de noviembre de 2013. Disponible en: http://www. arauca-arauca.gov.co/apc-aa-files/3630383435663734616 4393131343332/PROYECTO_PLAN_DESARROLLO_DE_ ARAUCA_2012_2015.pdf.

16. San Martín JL, Brathwaite-DO. La estrategia de gestión integrada para la prevención y control del dengue en la región de las Américas. Rev Panam Salud Pública. 2007;21:55-63. http://dx.doi.org/10.1590/S1020-49892007000100011

17. Alcaldía de Armenia. Plan de desarrollo 2012-2015. Armenia, un paraíso para invertir, vivir y disfrutar. Acuerdo No. 005, mayo 31 de 2012. Fecha de consulta: 15 de noviembre de 2013. Disponible en: http://www.armenia.gov. co/doc_usuarios/PDM2012.pdf.

18. Rodríguez CR. Estrategias para el control del dengue y del Aedes aegypti en las Américas. Rev Cubana Med Trop. 2002;5:189-201. 